\title{
The HSCT Unit
}

\author{
Walid Rasheed, Dietger W. Niederwieser, \\ and Mahmoud Aljurf
}

\subsection{Introduction}

HSCT is an advanced therapeutic intervention that is required for a number of malignant and nonmalignant medical conditions, often for critically ill patients. The establishment of an HSCT program requires the efforts of experienced and appropriately trained personnel to lead the program. Clearly, this also requires financial, legal, ethical, and other institutional support. For newly starting programs, it would be essential to identify minimal requirements for establishing an HSCT unit in order to optimize resource utilization as well as maintain safe patient care. While these minimal requirements also apply to wellestablished units, its structure helps to understand and implement additional steps for larger units which plan to offer additional transplant services and have access to more resources.

Approximately 20 years ago, the EBMT and the ISCT (International Society for Cellular Therapy) formed the Joint Accreditation CommitteeISCT and EBMT (JACIE) based on the FACT (Foundation for the Accreditation of Cellular Therapy) program. Efforts of these bodies have cul-

W. Rasheed $(\bowtie) \cdot$ M. Aljurf

King Faisal Specialist Hospital and Research Centre, Riyadh, Saudi Arabia

e-mail:wrasheed@kfshrc.edu.sa

D. W. Niederwieser

University of Leipzig, Leipzig, Germany minated in the establishment of standards related to HSCT and cellular therapies to assure quality and safety in the practice of HSCT. Although program accreditation with JACIE is not mandatory worldwide, these standards are very helpful as guidelines to understand requirements to establish an HSCT unit. Table 4.1 summarizes basic minimal requirements of an HSCT unit, which are discussed in more details in the following sections.

\subsection{Inpatient Unit}

The inpatient HSCT unit should have a minimum number of single-bedded rooms with isolation capability. The number and space of rooms should be adequate for the type and volume of transplant activity performed at the transplant center. These rooms must adhere to the standards of safety and comfort of patients in a tertiary care hospital facility. Every location or room should have a sink and tap for hand washing.

There needs to be a working station or room for nurses involved in patient care. A similar working space for physicians is required. Medical and nursing staff coverage should be available $24 \mathrm{~h}$ a day, including public holidays. The ratio of nurses to patient beds depends on the type and intensity of transplants being performed, e.g., autologous versus allogeneic, but generally, a ratio one nurse to three patients is reasonable. Emergency cart with drugs for resuscitation should be available in the inpatient unit. 
Table 4.1 HSCT unit minimal requirements

\begin{tabular}{|c|c|}
\hline Inpatient unit & - Clean single-bedded rooms with isolation capability \\
\hline $\begin{array}{l}\text { Ancillary medical } \\
\text { services }\end{array}$ & $\begin{array}{l}\text { - Intensive care unit } \\
\text { - Emergency room service } \\
\text { - Gastroenterology and pulmonary service }\end{array}$ \\
\hline Outpatient clinic & - Single patient examination rooms \\
\hline Blood bank & $\begin{array}{l}\text { - Twenty-four hour on-site blood bank service: } \mathrm{ABO} \text { typing and cross match, } \mathrm{RBC} \text {, and } \\
\text { platelets for transfusion } \\
\text { - Irradiation and leukocyte depletion of blood products }\end{array}$ \\
\hline Laboratory & $\begin{array}{l}\text { - Hematology cell count and chemistry lab } \\
\text { - Serology for viral screen } \\
\text { - Microbiology for basic bacterial and fungal cultures } \\
\text { - CMV PCR or antigenemia } \\
\text { - Access to CSA/tacrolimus levels }\end{array}$ \\
\hline HLA typing lab ${ }^{\mathrm{a}}$ & - Access to ASHI or similarly accredited HLA typing lab \\
\hline Stem cell collection & $\begin{array}{l}\text { - PBSC apheresis capability } \\
\text { - Bone marrow harvesting facility and expertise for matched sibling donor }\end{array}$ \\
\hline $\begin{array}{l}\text { Stem cell processing } \\
\text { facility }\end{array}$ & $\begin{array}{l}\text { - FACS CD34 enumeration } \\
\text { - Refrigerator for blood and bone marrow } \\
\text { - Controlled cryopreservation capability for freezing of autologous stem cell product } \\
\text { - Equipment and expertise to process ABO-mismatched cellular product }{ }^{\mathrm{a}}\end{array}$ \\
\hline Radiology & $\begin{array}{l}\text { - Routine X-ray radiology, ultrasound, and CT scanner } \\
\text { - Placement of central venous catheters }\end{array}$ \\
\hline Pharmacy & $\begin{array}{l}\text { - Availability of conditioning chemotherapy drugs } \\
\text { - Availability of antimicrobial agents (broad-spectrum antibiotics, antiviral, and antifungal } \\
\text { drugs) } \\
\text { - Availability of immunosuppressive agents for GVHD prophylaxis and treatment }{ }^{\mathrm{a}}\end{array}$ \\
\hline Human resources & $\begin{array}{l}\text { - Medical director: Licensed physician with adequate training and experience in HSCT } \\
\text { - Nursing staff with training in chemotherapy administration, infection control, and } \\
\text { handling of stem cell products } \\
\text { - Clinical laboratory director: Clinical pathology trained. } \\
\text { - Appropriately trained lab scientist and technicians } \\
\text { - Multidisciplinary medical staff (radiology, pathology, ICU, surgery, gastroenterology }{ }^{\mathrm{a}} \text {, } \\
\text { pulmonary }{ }^{\mathrm{a}} \text { ) }\end{array}$ \\
\hline Outcome database & - Monitor patient demographics, treatment, and outcomes (level I data reporting) \\
\hline Quality management & $\begin{array}{l}\text { - Written institutional protocols/guidelines } \\
\text { - Regular audits of various HSCT procedures and patient treatment outcomes } \\
\text { - System to detect errors or adverse events for corrective or preventative actions }\end{array}$ \\
\hline
\end{tabular}

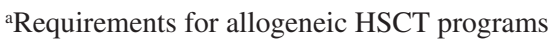

Infections, including bacterial, viral, or fungal infections, are potential significant complications in transplant recipient and may lead to significant morbidity and mortality. Therefore, HSCT units should have established measures for infection control. Guidelines for infection prevention and prophylaxis in HSCT patients, endorsed by several scientific organizations, are available and highly recommended to follow. HSCT recipients should be placed in single-patient rooms. Furthermore, at a minimum, standard precautions should be followed in all patients including hand hygiene and wearing of appropriate protective equipment (gloves, surgical masks or eye/face protection, gowns) during procedures/activities that are likely to generate splashes or spray of blood, body fluids, or secretions. Hand hygiene is essential, using alcohol-based hand rubs or washing with soap and water. In patients with suspected or proven of having an infection, additional precautions are required accordingly, e.g., airborne, droplet, or contact isolation. HSCT units should be cleaned at least daily with special attention to dust control. During building construction, intensified mold control measures are required, and a multidisciplinary team should be involved.

Other important infection control measures include well-sealed rooms, positive pressure 
differential between patient rooms and the hallway, self-closing doors, more than 12 air exchanges per hour, and continuous pressure monitoring. HEPA (high efficiency particulate air) filters have shown efficacy in providing protection against acquisition of fungal infections in immune-compromised hematology patients, including HSCT patients, and during hospital construction or renovation works. While HEPA filters are not absolutely required as a minimal requirement in newly established centers with less complicated transplant activities, they are certainly preferred and highly recommended as newly established centers expand their activities to include more complicated (especially allogeneic) transplant activities.

There is no agreed upon minimum number of transplants to be performed in a program. However, to ensure continuing proficiency in a transplant program, the ASBMT recommends for programs performing only one type of HSCT (autologous or allogeneic), at least ten transplants of that type are to be performed per annum; programs performing both allogeneic and autologous transplantations should perform a minimum of ten transplants of each kind per annum.

\subsection{Ancillary Medical Services}

HSCT patients often require other medical specialties involvement in their complicated care. This includes the risk of developing lifethreatening infections or other post transplant complications, hence the importance of having access to emergency room as well as intensive care services at the same tertiary care hospital facility where transplant program is being established. Intensive care units should have the ability of providing inotropic support, respiratory support (including mechanical ventilation) as well as renal replacement (hemodialysis) if required.

Input from infectious disease physicians can be valuable in HSCT patients who are at risk of a multitude of opportunistic and potentially lifethreatening infections; this is especially important for programs that perform allogeneic transplants. Availability of gastroenterology specialist with endoscopy services is critical for allogeneic programs, as often diagnostic endoscopy is required to differentiate GVHD from other etiologies of gastrointestinal complications. Similarly, pulmonary medicine service with access to diagnostic bronchoscopies is required for such patients with pulmonary abnormalities.

HSCT programs that perform transplants using radiotherapy as part of conditioning regimen (total body irradiation) should have available radiation oncology service on site. The radiation oncology team, including the radiation oncologist and physicist, should have adequate training in the technique of total body irradiation and appropriate equipment, and procedures must be in place to deliver successful and safe radiation component of these conditioning regimen.

\subsection{Outpatient Unit}

HSCT patients attend to the outpatient unit, both for pretransplant assessment and work-up and post transplant follow-up and management. Single patient examination rooms are a minimal requirement for the outpatient service of the program. These rooms should be adequately equipped to allow clinical assessment of patients. It is important to implement infection control measures to minimize risk of transmitting infections, including hand hygiene measures and availability of appropriate room to isolate patients who are identified to be potentially infectious to others, e.g., due to herpes zoster infection. A dedicated infusion area would be ideal as transplant recipients often require IV fluid and electrolyte replacement or blood product administration.

\subsection{Blood Bank}

Availability of blood banking services is a critical component of a successful transplant program. A 24-h on-site blood banking service is required for ABO typing, cross match, and urgent supply of red blood cells and platelets for transfusion. Meeting minimal standard criteria according to 
recognized international blood bank societies such as the American Association of Blood Banks (AABB) or equivalent is important. Blood bank staff, including blood bank director, scientists, and technicians should be adequately qualified and trained in blood banking procedures.

Transplant recipients are severely immunecompromised and are at risk of transfusionassociated GVHD, caused by unrestricted proliferation of donor lymphocytes in the immune-compromised host. Hence, it is critical that transplant recipients receive irradiated blood products to prevent this complication. The use of leukocyte-depleted blood products is recommended to reduce the risk of HLA alloimmunization in the multiply transfused hematology patients, as well as to reduce the incidence of transfusion reactions. In allogeneic programs, clear documented pathways for transfusion support in cases of ABO mismatch should be available for both blood bank and clinical staff as guidance.

\subsection{Laboratory}

A 24-h on-site hematology cell count and basic chemistry lab are required. Furthermore, microbiology laboratory service is essential in the clinical management of transplant recipients, including routine bacterial and fungal cultures of various patient specimens. Serology screening for relevant viral and bacterial infections is also required for pretransplant work-up of recipients as well as donor screening. For allogeneic transplant recipients, monitoring for cytomegalovirus (CMV) reactivation is essential, and results must be available in a timely manner to allow therapeutic intervention; both molecular technique by quantitative PCR (preferable) and antigenemia method are acceptable. In the allogeneic setting, monitoring drug levels, e.g., cyclosporine or tacrolimus, is required, and same-day service is recommended to allow interventions aiming at keeping levels of these important drugs within the target therapeutic range.

\subsection{HLA Typing Lab}

Access to HLA typing laboratory is mandatory for allogeneic programs. Such service can be available on-site or alternatively provided in reference laboratory. JACIE standards state that clinical programs performing allogeneic transplantation shall use HLA testing laboratories that are capable of carrying out DNA-based intermediate and high-resolution HLA typing and are appropriately accredited by the American Society for Histocompatibility and Immunogenetics (ASHI), European Federation for Immunogenetics (EFI), or other accrediting organizations providing histocompatibility services appropriate for hematopoietic cellular therapy transplant patients.

\subsection{Stem Cell Collection}

Access to peripheral blood stem cell apheresis service on-site is a minimal requirement in each program. This is often part of the blood bank service or alternatively under the administration of the clinical program. Having at least two cell separators would be beneficial, as the second cell separator would be a backup in situations of unexpected machine faults and for routine servicing. Daily operation of apheresis facility requires appropriately trained and experienced nursing staff and a medical director with adequate qualification and experience in clinical and laboratory aspects of the apheresis procedure. Institutional written protocols and policies covering all aspects of apheresis procedure should be available for guidance. JACIE standards require a minimum average of ten cellular therapy products collected by apheresis per year for program accreditation.

A bone marrow stem cell source is sometimes recommended for better patient outcome, e.g., patients with bone marrow failure. Programs performing allogeneic transplants for such indication should have a bone marrow harvest facility on-site. This requires convenient and easy access to surgical operating room with anesthesia service. Appropriate equipment for the bone mar- 
row harvest procedure are required. Physicians with adequate training and experience in bone marrow harvesting are crucial to perform the procedure successfully.

\subsection{Stem Cell Processing Facility}

The stem cell processing facility requires a designated area, usually within the laboratory. It should be appropriately equipped for the processing of various stem cell products depending on the types of transplants performed and the size of the program. Availability of flow cytometry for the enumeration of CD34 cell count is mandatory. Controlled cryopreservation capability, using liquid nitrogen, for freezing of autologous stem cell product is essential. This may also be used in allogeneic sibling products. Standard quality control measures, including systems to closely monitor and record the temperature in all freezes and refrigerators, are critical. Allogeneic programs should have appropriate equipment and expertise on-site for the timely and safe processing of ABOmismatched stem cell products as required, including the need to perform red cell or plasma depletion procedures when indicated. The processing facility should be operated by adequately trained staff, including scientist, technicians, and a medical director. Written standard operating procedures explaining all aspects of stem cell processing performed at the facility are required.

\subsection{Radiology}

Standard routine (X-ray), ultrasound, and computed tomography (CT scan) imaging services are the minimal requirements and should be available on site for the routine diagnostic imaging. Availability of magnetic resonance imaging (MRI) is preferred, as it is often useful in the diagnosis of specific clinical conditions relevant to stem cell transplant recipients, such as iron overload, CNS infections, and posterior reversible encephalopathy syndrome (PRES) related to calcineurin inhibitor toxicity. Placement of central venous catheters in transplant recipients is obviously required in each program. Depending on the institutional setting, this service may be provided by various hospital services; often this is done by the radiology service under ultrasound guidance. Having well trained and experienced interventional radiologist to perform this procedure is crucial for the safety of patients.

\subsection{Pharmacy}

Pharmacy services are essential in each HSCT program. Availability of conditioning chemotherapy agents is clearly required; specific drugs depend on the type and complexity of transplant procedures performed in each program. Commonly used agents in conditioning regimens include BU, CY, FLU, and MEL. ATG may also be required in the allogeneic setting (e.g., in aplastic anemia) and requires special attention and training by nursing, pharmaceutical, and medical staff in relation to its administration.

Broad-spectrum antibiotics should be available for urgent use as required in transplant recipient. Likewise, access to antiviral and antifungal agents is important for both prophylaxis and treatment. Allogeneic programs should also have access to immunosuppressive drugs used for GVHD prophylaxis such as CSA, MTX, and TAC.

A trained pharmacist is crucial for the HSCT program. The pharmacist should review all conditioning chemotherapy protocols and ensure appropriate dispensing and administration of cytotoxic agents.

\subsection{Staffing and Human Resources}

Appropriately trained and experienced medical and nursing staffs are crucial for the HSCT program. The clinical medical director of the program should be a licensed physician (specialty certification in hematology, oncol- 
ogy, or immunology) with adequate training at a BMT program. A minimal BMT training duration of 6-12 months is suggested. JAICIE standards indicate that the clinical program director shall have 2 years of experience as an attending physician responsible for the direct clinical management of HSCT patients in the inpatient and outpatient settings. A minimum of one (1) additional attending transplant physician is required in the program.

The success of a transplant program relies heavily on the presence of appropriately trained and experienced nursing staff. This includes training in chemotherapy administration, infection control, management of neutropenic patients, and handling of stem cell products.

Other important staff includes appropriately trained and experienced personnel in the laboratory (including laboratory director, scientist, and technicians), trained pharmacist, as well as medical professionals of ancillary medical services. Continuous education activities are required for all healthcare professionals involved in the management of HSCT patients.

\subsection{Institutional Database and Data Manager}

Monitoring patient demographics, treatment details, and outcomes is an essential minimal requirement. Each program should keep complete and accurate patient records, and a database containing relevant patient data should be established and regularly maintained. Appropriate patient consent needs to be obtained for such database. An example of the minimal data required to be obtained on each transplant patient is the information required in the CIBMTR or EBMT mid A forms. Having a data manager in a transplant program to initiate and maintain this institutional transplant database is highly recommended. Often data managers have nursing background with experience in stem cell transplantation. Attending training data management courses during international meetings or through links with other experienced and well-established programs would be valuable.

\subsection{Quality Control}

The JACIE standards require that all essential clinical collection and processing facilities in the transplant center evaluate and report patient outcomes. Regular audits of various HSCT procedures and patient treatment outcomes are required. Essentially, a system is required to be in place to detect errors/adverse events, so that these can be evaluated in order to implement preventative measures to minimize the risk of recurrence of these incidents. Furthermore, each program should have written institutional clinical protocols in relation to the various aspects of the transplant patient care to standardize practice. Likewise, stem cell collection and processing facilities should have standard operating procedures that serve as a guidance for all staff to follow to enhance patient's safety. Access to or relationship with experienced HSCT program is often very helpful and highly recommended via shared protocols/telemedicine and/or web-based conferencing.

\subsection{Transplant Coordinator}

HSCT is a complex therapeutic intervention, and coordination of the pretransplant, transplant, and post transplant patient care is important. A transplant coordinator can play pivotal role in this context, acting as a facilitator, educator, as well as a point of contact for the patient and their families. Transplant coordinators ensure the smooth and safe running of the HSCT service starting from scheduling and arranging pretransplant work-up of patient and planning the roadmap for the transplant recipient with continued involvement and education of the patients and their families until the time of admission. Furthermore, transplant coordinator would play a significant role in the coordination of post HSCT follow-up and care in clinics. For allo-HSCT, the transplant coordinator would be very valuable in arranging donor search starting from HLA typing of the recipient and his/her family members, in addition to initiating and following a search for unrelated donor in national or international registries. 
The transplant coordinator involvement may extend to organizing the logistics of getting the stem cells from the donor from the donor center where the recipient may be in another health facility (national or international). Moreover, transplant coordinators will often lead the HSCT team weekly planning meetings and discussions with the arrangement of the HSCT waiting list. Typically, transplant coordinators have nursing background with significant experience in stem cell transplantation.

\section{Key Points}

- The inpatients unit should have singlebedded rooms with isolation capabilities. Single outpatient examination rooms are also required.

- Laboratory, blood bank, and pharmacy services are critical to the success of HSCT programs.

- Stem cell collection and processing capabilities are minimal requirements for any HSCT program; the level of such capabilities depends on the type and complexity of HSCT performed in each center.

- Ancillary medical services are essential components of successful HSCT programs, including intensive care and emergency and radiology services. Additional medical services are required in allogeneic programs.

- Appropriately trained and experienced staff (medical, nursing, laboratory, pharmacy) are crucial for the HSCT program.

- Monitoring patient characteristics and transplant outcomes is essential.

- A local quality control system is required in all aspects involved in the HSCT procedure.

- Having a data manager for the HSCT program, to initiate and maintain institutional minimal transplant data base is highly recommended.
- Transplant coordinators play pivotal role the management of HSCT patients, starting from pre SCT work up, right through post transplant care.

\section{Recommended References}

Booth GS, Gehrie EA, Bolan CD, Savani BN. Clinical guide to ABO-incompatible allogeneic stem cell transplantation. Biol Blood Marrow Transplant. 2013;19:1152-8.

Chang CC, Ananda-Rajah M, Belcastro A, et al. Consensus guidelines for implementation of quality processes to prevent invasive fungal disease and enhanced surveillance measures during hospital building works, 2014. Intern Med J. 2014;44:1389-97.

Crysandt M, Yakoub-Agha I, Reiß P, et al. How to build an allogeneic hematopoietic cell transplant unit in 2016: proposal for a practical framework. Curr Res Trans1 Med. 2017;65:149-54.

Daniele N, Scerpa MC, Rossi C, et al. The processing of stem cell concentrates from the bone marrow in ABO-incompatible transplants: how and when. Blood Transfus. 2014;12:150-8.

FACT/JACIE. FACT-JACIE 7th edition standards; 2018. http://www.jacie.org/.

Hahn T, Cummings KM, Michalek AM, et al. Efficacy of high-efficiency particulate air filtration in preventing aspergillosis in immunocompromised patients with hematologic malignancies. Infect Control Hosp Epidemiol. 2002;23:525-31.

Krüger WH, Zöllner B, Kaulfers PM, Zander AR. Effective protection of allogeneic stem cell recipients against Aspergillosis by HEPA air filtration during a period of construction--a prospective survey. J Hematother Stem Cell Res. 2003;12:301-7.

Leemhuis T, Padley D, Keever-Taylor C, et al. Essential requirements for setting up a stem cell processing laboratory. Bone Marrow Transplant. 2014;49:1098-105.

Napier A, Williamson LM. British Committee for Standards in Haematology, Blood Transfusion Task Force, et al. Guidelines on the clinical use of leucocyte - depleted blood components. Transfusion Med. 1998;81:59-71.

Phillip G, Armitage J, Bearman S. American Society for Blood and Marrow Transplantation guidelines for clinical centers. Biol Blood Marrow Transplant. 1995;1:54-5.

Tomblyn M, Chiller T, Einsele H, et al. Guidelines for preventing infectious complications among hematopoietic cell transplantation recipients: a global perspective. Biol Blood Marrow Transplant. 2009;15:1143-238.

Treleaven J, Gennery A, Marsh J, et al. Guidelines on the use of irradiated blood components prepared by the British committee for standards in haematology blood transfusion task force. Br J Haematol. 2011;152:35-51. 
Open Access This chapter is licensed under the terms of the Creative Commons Attribution 4.0 International License (http://creativecommons.org/licenses/by/4.0/), which permits use, sharing, adaptation, distribution and reproduction in any medium or format, as long as you give appropriate credit to the original author(s) and the source, provide a link to the Creative Commons license and indicate if changes were made.

The images or other third party material in this chapter are included in the chapter's Creative Commons license, unless indicated otherwise in a credit line to the material. If material is not included in the chapter's Creative Commons license and your intended use is not permitted by statutory regulation or exceeds the permitted use, you will need to obtain permission directly from the copyright holder.

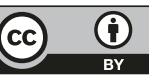

